\title{
DYNAMICAL SYSTEMS THAT COMPUTE BALANCED REALIZATIONS AND THE SINGULAR VALUE DECOMPOSITION*
}

\author{
U. HELMKE ${ }^{\dagger}$, J. B. MOORE $\ddagger$, AND J. E. PERKINS ${ }^{\ddagger}$
}

\begin{abstract}
The tasks of finding balanced realizations in systems theory and the singular value decomposition (SVD) of matrix theory are accomplished by finding the limiting solutions of differential equations. Several alternative sets of equations and their convergence properties are investigated. The dynamical systems for these tasks generate flows on the space of realizations that leave the transfer functions invariant. They are termed isodynamical flows. Isodynamical flows are generalizations of isospectral flows on matrices. These flows evolve on the actual system matrices and thus remove the need for considering coordinate transformation matrices. The methods are motivated by the power of parallel processing and the ability of a differential equations approach to tackle time-varying or adaptive tasks.
\end{abstract}

Key words. balanced realization, singular value decomposition, gradient flow, differential equation, dynamical systems

AMS subject classifications. 93B20, 93B40, 15A18, 65F 15

1. Introduction. In current practice, the problems of finding a balanced realization for a linear control system, as well as achieving an SVD of a matrix are solved using algebraic matrix manipulations, implemented in standard computer programs. Balanced realizations are a useful tool in systems theory to increase numerical robustness, and they allow a sensible model order reduction to be performed. This operation has been widely studied $[10],[11]$ and computation methods have been described. Certainly, these methods are widely used, reliable, and well understood. On the other hand, recent advances in neural network theory and associative memories have shown that gradient-type algorithms can lead to effective and fast methods for algebraic tasks such as principle component analysis. This latter task is equivalent to the SVD. It follows that gradient flows can be an effective tool for SVD, although the full possibilities and limitations of this approach are not yet fully clear.

Brockett [1], again motivated by the renewed interest in neural networks, parallel processing, and analog computing, has also shown that other linear algebra and combinatorial problems can be solved in terms of the limiting solutions of ordinary differential equations (ODEs) that are gradient flows on orthogonal matrices. In [2] a systematic approach to balanced realizations of linear systems was developed, which treats balanced realizations as the global minima of objective functions, defined on the set of all realizations of a given transfer function. Aspects of this work are generalized in [3] for the task of finding an SVD using gradient flows on unitary matrices. In an earlier paper [4], it is shown how certain types of balancing problems can be solved using gradient flows on positive definite matrices with an exponential rate of convergence. Such algorithms are possibly suitable for application to time-varying systems [5].

In this paper, a systematic attempt is made to construct and analyze dynamical systems that are capable of achieving balancing or the SVD. Based on the cost

* Received by the editors November 25, 1991; accepted for publication (in revised form) September 23, 1992. This work was partially supported by Boeing (BCAC).

$\dagger$ Department of Mathematics, University of Regensburg, 93040 Regensburg, Germany (helmke@vax 1.rz.uni-regensburg. 2400 .de).

$\ddagger$ Department of Systems Engineering, Research School of Physical Sciences, Australian National University, GPO Box 4, Canberra ACT 2601 (janeøoberon.dsto.gov,au, jbom1010rsphy1.anu.edu.au). 
function approach developed in [2], we propose several different gradient flows that solve the problem of finding a balanced realization, given an initial system realization. Each of these equations has an exponential rate of convergence and we compare their respective rates. It is envisaged that for particular applications there will be one gradient flow that will give a better convergence rate than other algorithms. First we review the linear and quadratic gradient flows of [4] that evolve on $P=T^{\prime} T>0$, where $T$ is the state space transformation matrix that gives the balanced realization. The next solution method we consider are differential equations that evolve on the actual transformation matrix $T$. This solution method is of interest because it circumvents the need to find $T$ given $P=T^{\prime} T$.

Next we propose alternative ODEs that solve the balanced realization problem. These differential equations, termed isodynamical flows, evolve on the actual system matrices $(A, B, C)$ rather than having the intermediate step of transformation matrices. They have the obvious advantage of immediacy as well as giving a clearer indication as to how the system is evolving. This is the first time a direct method to compute balanced realizations, without computing any balancing transformations, has been given. The class of all isodynamical flows can be viewed as a generalization of the isospectral flows, studied in matrix theory, as in [1], [3], [6], [7], [9], and their references.

In $\S 2$ gradient flows that give the transformation matrices for balanced realizations are studied, and in $\S 3$ related $O D E s$ are developed for a direct evolution of the system matrices. In $\S 4$ flows achieving the SVD of a matrix are studied, and in $\S 5$ conclusions are drawn. The Appendix summarizes important results about gradient flows on manifolds.

2. Gradient flows for balancing transformations. In this section we consider the problem of computing balancing coordinate transformations via differential equations. While a part of this problem has been already considered in [4], we review some of the material developed in [4] and emphasize some new points as well.

We consider linear dynamical systems in continuous or discrete time

$$
\left\{\begin{array}{l}
\dot{x}(t)=A x(t)+B u(t) \\
y(t)=C x(t)
\end{array}\right\} \text { or } \quad\left\{\begin{array}{c}
x_{k+1}=A x_{k}+B u_{k} \\
y_{k}=C x_{k}
\end{array}\right\}
$$

defined by the system matrices $(A, B, C) \in \Re^{n \times n} \times \Re^{n \times m} \times \Re^{p \times n}$. Such a system is called asymptotically stable if the eigenvalues of $A$ are in the open complex left half-plane or in the open unit disc, respectively. For any asymptotically stable system $(\mathrm{A}, \mathrm{B}, \mathrm{C})$, the controllability and observability gramians $W_{c}$ and $W_{o}$ are, respectively, defined in discrete time and continuous time by the symmetric matrices

$$
\begin{array}{r}
W_{c}=\sum_{k=0}^{\infty} A^{k} B B^{\prime} A^{\prime k}, \quad W_{o}=\sum_{k=0}^{\infty} A^{\prime k} C^{\prime} C A^{k} \\
W_{c}=\int_{0}^{\infty} e^{A t} B B^{\prime} e^{t A^{\prime}} \mathrm{dt}, \quad W_{o}=\int_{0}^{\infty} e^{t A^{\prime}} C^{\prime} C e^{A t} \mathrm{dt} .
\end{array}
$$

For unstable systems the controllability and observability gramians are likewise defined by finite sums or integrals rather than by the above infinite sums or integrals. In the following we will assume asymptotic stability of $A$; however, all results hold mutatis mutandis in the unstable case using finite gramians. To emphasize the dependence of the gramians on $(A, B, C)$, we also write $W_{c}(A, B)$ and $W_{o}(A, C)$ for the controllability and observability gramians of $(A, B, C)$. 
In the sequel we fix an initial asymptotically stable controllable and observable realization $(A, B, C) \in \Re^{n \times n} \times \Re^{n \times m} \times \Re^{p \times n}$ of a given transfer function $G(s)=$ $C(s I-A)^{-1} B \in \Re(s)^{p \times m}$. Thus, by Kalman's realization theorem, see, e.g., Kailath [8], all other minimal realizations of $G(s)$ are of the form $\left(T A T^{-1}, T B, C T^{-1}\right)$ for a uniquely determined invertible coordinate transformation $T$.

Any linear change of coordinates in the state space $\Re^{n}$ by an invertible transformation $T \in G L(n, \Re)$ changes the realization according to $(A, B, C) \mapsto\left(T A T^{-1}\right.$, $T B, C T^{-1}$ ) and thus transforms the gramians via

$$
W_{c} \mapsto T W_{c} T^{\prime}, \quad W_{o} \mapsto\left(T^{\prime}\right)^{-1} W_{\mathrm{o}} T^{-1} .
$$

We call a state space representation $(A, B, C)$ of the transfer function balanced if $W_{c}=W_{o}$. This is more general than the usual definition of balanced realizations, (see Moore [10]), which requires that $W_{c}=W_{o}=$ diagonal. In this case we refer to $(A, B, C)$ as a diagonal balanced realization, which is thus one particular realization of our class of balanced realizations.

To obtain a quantitative measure of how the gramians change for the various realizations of a transfer function, we consider the function

$$
\begin{aligned}
\Phi(T) & =\operatorname{tr}\left(W_{c}\left(T A T^{-1}, T B\right)+W_{o}\left(T A T^{-1}, C T^{-1}\right)\right) \\
& =\operatorname{tr}\left(T W_{c} T^{\prime}+\left(T^{\prime}\right)^{-1} W_{o} T^{-1}\right) \\
& =\operatorname{tr}\left(W_{c} T^{\prime} T+W_{o}\left(T^{\prime} T\right)^{-1}\right) \\
& =\operatorname{tr}\left(W_{c} P+W_{o} P^{-1}\right)
\end{aligned}
$$

with

$$
P=T^{\prime} T
$$

Note that $\Phi(T)$ is the sum of the eigenvalues of the controllability and observability gramians of $\left(T A T^{-1}, T B, C T^{-1}\right)$ and is thus a crude numerical measure for the controllability and observability of $\left(T A T^{-1}, T B, C T^{-1}\right)$.

2.1. Balancing flows of positive definite matrices. Let $\mathcal{P}(n)$ denote the set of positive definite real symmetric $n \times n$ matrices $P=P^{\prime}>0 . \mathcal{P}(n)$ is an open, convex subset of the set of all symmetric $n \times n$ matrices and is diffeomorphic to Euclidean space $\Re^{(1 / 2) n(n+1)}$. By (2.3) we are led to study the function

$$
\phi: \mathcal{P}(n) \rightarrow \Re, \quad \phi(P)=\operatorname{tr}\left(W_{c} P+W_{o} P^{-1}\right) .
$$

For a proof of the following results we refer to [4] and [9].

LEMMA 2.1 ([4], [9]). Let $W_{c}, W_{o}$ be the controllability and observability gramians (2.1) of an asymptotically stable minimal realization $(A, B, C)$. Then the function $\phi: \mathcal{P}(n) \rightarrow \Re, \phi(P)=\operatorname{tr}\left(W_{c} P+W_{o} P^{-1}\right)$, defined on the set $\mathcal{P}(n)$ of positive definite symmetric matrices, has compact sublevel sets, i.e., for all $a \in \Re$ then $\left\{P \in \mathcal{P}(n) \mid \operatorname{tr}\left(W_{c} P+W_{o} P^{-1}\right) \leq a\right\}$ is a compact subset of $\mathcal{P}(n)$. In particular, there exists a minimizing $P=P^{\prime}>0$ for the function $\phi: \mathcal{P}(n) \rightarrow \Re$ defined by (2.5).

While Lemma 2.1 establishes the existence of a minimizing $P_{\infty}=P_{\infty}^{\prime}>0$ for the function (2.5), Theorem 2.2 provides a more constructive approach towards finding the minimum by showing that it is the global attractor for the gradient flow on $\mathcal{P}(n)$.

THEOREM 2.2. Linear index gradient flow ([4]). Let $W_{c}, W_{o}$ denote the controllability and observability gramians (2.1) of an asymptotically stable, controllable, and observable realization $(A, B, C)$. 
(a) There exists a unique $P_{\infty}=P_{\infty}^{\prime}>0$, which minimizes $\phi: \mathcal{P}(n) \rightarrow \Re, \phi(P)=$ $\operatorname{tr}\left(W_{c} P+W_{o} P^{-1}\right)$, and $T_{\infty}=P_{\infty}^{1 / 2}$ is a balancing transformation for $(A, B, C)$. This minimum is given by

$$
P_{\infty}=W_{c}^{-1 / 2}\left(W_{c}^{1 / 2} W_{o} W_{c}^{1 / 2}\right)^{1 / 2} W_{c}^{-1 / 2}
$$

(b) The gradient flow $\dot{P}(t)=-\nabla \phi(P(t))$ of $\phi: \mathcal{P}(n) \rightarrow \Re$ is given by

$$
\dot{P}=P^{-1} W_{o} P^{-1}-W_{c} .
$$

For every initial condition $P_{o}=P_{o}^{\prime}>0, P(t)$ exists for all $t \geq 0$ and converges exponentially fast to $P_{\infty}$ as $t \rightarrow \infty$ with a lower bound for the rate of exponential convergence given by

$$
\rho \geq 2 \frac{\lambda_{\min }\left(W_{c}\right)^{3 / 2}}{\lambda_{\max }\left(W_{o}\right)^{1 / 2}}
$$

where $\lambda_{\min }(A)$ respectively $\lambda_{\max }(A)$ denote the smallest, respectively largest, eigenvalue of $A$.

In the sequel we refer to (2.6) as the linear index gradient flow. Instead of minimizing $\phi(P)$, we might as well consider the minimization problem for the quadratic index function

$$
\Psi(P)=\operatorname{tr}\left(\left(W_{c} P\right)^{2}+\left(W_{o} P^{-1}\right)^{2}\right)
$$

over all positive definite symmetric matrices $P=P^{\prime}>0$.

Since, for $P=T^{\prime} T, \Psi(P)$ is equal to $\operatorname{tr}\left[\left(T W_{c} T^{\prime}\right)^{2}+\left(\left(T^{\prime}\right)^{-1} W_{o} T^{-1}\right)^{2}\right]$, the minimization problem for $(2.8)$ is equivalent to minimizing $\operatorname{tr}\left[\left(W_{c}\left(T A T^{-1}, T B\right)\right)^{2}+\right.$ $\left.\left(W_{\circ}\left(T A T^{-1}, C T^{-1}\right)\right)^{2}\right]$ over the set of all realizations $\left(T A T^{-1}, T B, C T^{-1}\right)$ of a given transfer function $G(s)=C(s I-A)^{-1} B$. Thus $\Psi(P)$ is the sum of the squared eigenvalues of the controllability and observability gramians of $\left(T A T^{-1}, T B, C T^{-1}\right)$. Note also that

$$
\operatorname{tr}\left[\left(T W_{c} T^{\prime}\right)^{2}+\left(\left(T^{\prime}\right)^{-1} W_{o} T^{-1}\right)^{2}\right]=\left\|T W_{c} T^{\prime}-\left(T^{\prime}\right)^{-1} W_{o} T^{-1}\right\|^{2}+2 \operatorname{tr}\left[W_{c} W_{o}\right] .
$$

Thus minimizing this quadratic index function is equivalent to minimizing the least square distance $\left\|T W_{c} T^{\prime}-\left(T^{\prime}\right)^{-1} W_{o} T^{-1}\right\|^{2}$.

THEOREM 2.3. Quadratic index gradient flow ([4]). Under the same hypotheses as for Theorem 2.2, we have:

(a)

$$
P_{\infty}=W_{c}^{-1 / 2}\left(W_{c}^{1 / 2} W_{\mathrm{o}} W_{c}^{1 / 2}\right)^{1 / 2} W_{c}^{-1 / 2}
$$

is the uniquely determined $P \in \mathcal{P}(n)$ which minimizes $\Psi: \mathcal{P}(n) \rightarrow \Re$ and $T_{\infty}=P_{\infty}^{1 / 2}$ is a balancing transformation for $(A, B, C)$.

(b) The gradient flow $\dot{P}(t)=-\nabla \Psi(P(t))$ on $\mathcal{P}(n)$ is

$$
\dot{P}=2 P^{-1} W_{o} P^{-1} W_{o} P^{-1}-2 W_{c} P W_{c} \text {. }
$$

For all initial conditions $P_{o}=P_{o}^{\prime}>0$, the solution $P(t)$ of $(2.9)$ exists for all $t \geq 0$ and converges exponentially to $P_{\infty}$. A lower bound on the rate of exponential convergence is

$$
\rho>4 \lambda_{\min }\left(W_{c}\right)^{2} .
$$


We refer to (2.9) as quadratic index gradient flow. The above results show that both algorithms converge exponentially fast to $P_{\infty}$. Both algorithms are rather slow if the smallest singular value of $W_{c}$ is near to zero, i.e., if the system is nearly uncontrollable. In contrast to this behaviour, (2.7) shows that the convergence of the linear index flow becomes relatively fast if $\lambda_{\max }\left(W_{o}\right)$; that is, the 2-norm $\left\|W_{o}\right\|_{2}$ of the observability gramian is small. Similarly, the bound (2.10) for the rate of convergence of the quadratic index flow is independent of $W_{o}$ and therefore we expect a certain amount of robustness of our algorithms in the case where the observability properties of the system are poor.

In general, the quadratic index flow seems to behave better than the linear index flow, at least if the smallest singular value of the associated Hankel operator of $(A, B, C)$ is greater than $\frac{1}{2}$, i.e., if $\lambda_{\min }\left(W_{o} W_{c}\right)>\frac{1}{4}$. This is supported by the following simulations.

Simulations. The following simulations show the exponential convergence of the diagonal elements of $P$ towards the solution matrix $P_{\infty}$ and illustrate what might affect the convergence rate. In Figs. $1(\mathrm{a})-(\mathrm{c})$ we have

$$
W_{\circ}=W_{3}=\left[\begin{array}{llll}
7 & 4 & 4 & 3 \\
4 & 4 & 2 & 2 \\
4 & 2 & 4 & 1 \\
3 & 2 & 1 & 5
\end{array}\right] \text { and } W_{c}=W_{4}=\left[\begin{array}{cccc}
5 & 2 & 0 & 3 \\
2 & 7 & -1 & -1 \\
0 & -1 & 5 & 2 \\
3 & -1 & 2 & 6
\end{array}\right]
$$

so that $\lambda_{\min }\left(W_{o} W_{c}\right) \approx 1.7142>\frac{1}{4}$. Figure $1(\mathrm{a})$ concerns the linear index flow, while Fig. 1(b) shows the evolution of the quadratic index flow, both using $P(0)=P_{1}$, where

$$
P(0)=P_{1}=\left[\begin{array}{llll}
1 & 0 & 0 & 0 \\
0 & 1 & 0 & 0 \\
0 & 0 & 1 & 0 \\
0 & 0 & 0 & 1
\end{array}\right], \quad P(0)=P_{2}=\left[\begin{array}{llll}
2 & 1 & 0 & 0 \\
1 & 2 & 1 & 0 \\
0 & 1 & 2 & 1 \\
0 & 0 & 1 & 2
\end{array}\right]
$$

Figure 1(c) shows the evolution of both algorithms with a starting value of $P(0)=P_{2}$. These three simulations demonstrate that the quadratic algorithm converges more rapidly than the linear algorithm when $\lambda_{\min }\left(W_{o} W_{c}\right)>\frac{1}{4}$. This rapid convergence rate is achieved at the expense of twice the number of matrix multiplications in calculating the gradient.

In Fig. 1(d),

$$
W_{o}=W_{1}=\left[\begin{array}{llll}
7 & 4 & 4 & 3 \\
4 & 4 & 2 & 2 \\
4 & 2 & 4 & 1 \\
3 & 2 & 1 & 3
\end{array}\right] \text { and } W_{c}=W_{2}=\left[\begin{array}{cccc}
5 & 4 & 0 & 3 \\
4 & 7 & -1 & -1 \\
0 & -1 & 5 & 2 \\
3 & -1 & 2 & 6
\end{array}\right]
$$

so that $\lambda_{\min }\left(W_{o} W_{c}\right) \approx 0.207<\frac{1}{4}$. Figure $1(\mathrm{~d})$ compares the linear index flow behaviour with that of the quadratic index flow for $P(0)=P_{1}$. This simulation demonstrates that the linear algorithm does not necessarily converge more rapidly than the quadratic algorithm when $\lambda_{\min }\left(W_{o} W_{c}\right)<\frac{1}{4}$, because the bounds on convergence rates are conservative.

2.2. Gradient flows for balancing transformations. In the previous section we studied gradient flows that converged to $P_{\infty}=T_{\infty}^{2}$, where $T_{\infty}$ is the unique symmetric positive definite balancing transformation for a given asymptotically stable 


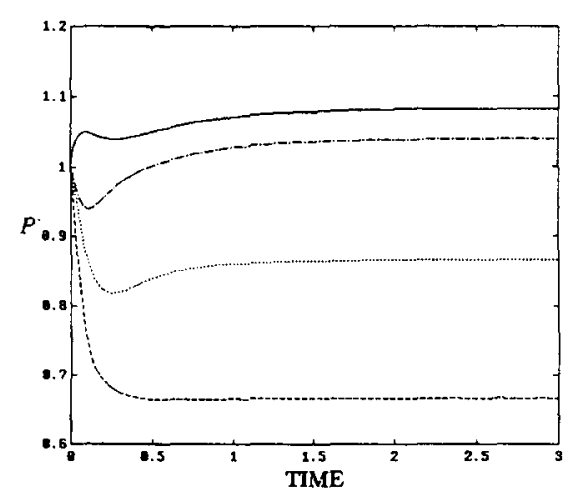

(a)

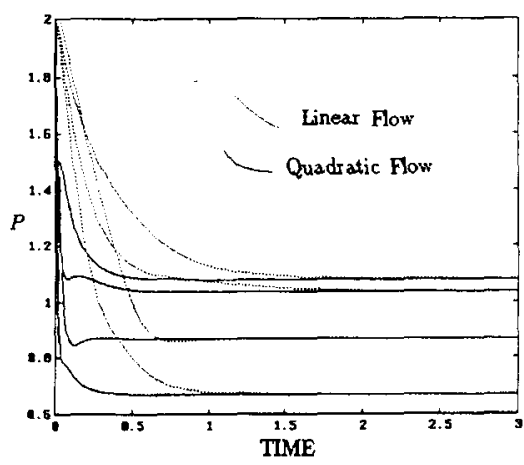

(c)

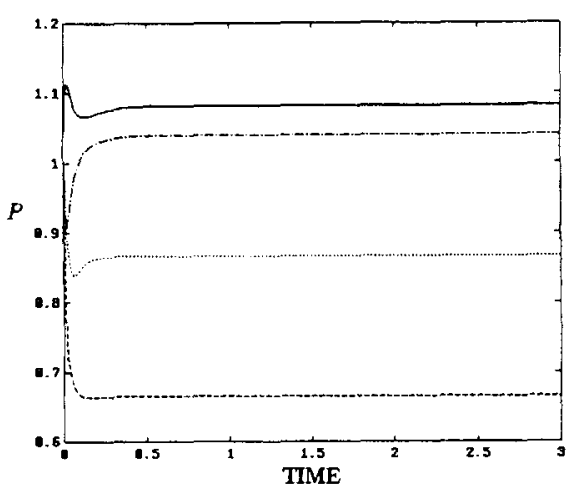

(b)

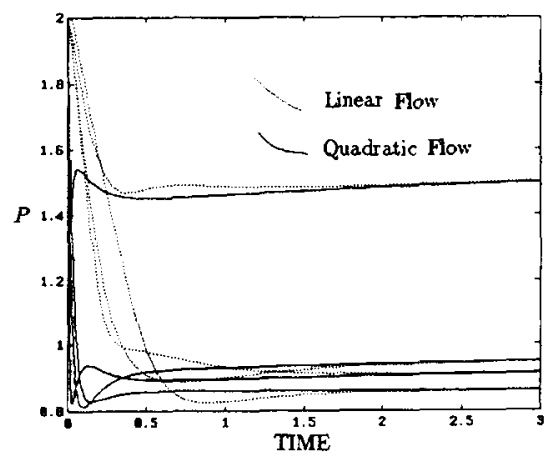

(d)

FIG. 1. Comparison of linear and quadratic flows on $P(t)$. (a) Linear index flow when $\lambda_{\min }\left(W_{o} W_{c}\right)>\frac{1}{4}$. (b) Quadratic index flows when $\lambda_{\min }\left(W_{o} W_{c}\right)>\frac{1}{4}$. (c) Linear and quadratic flows when $\lambda_{\min }\left(W_{o} W_{c}\right)>\frac{1}{4}$. (d) Linear and quadratic flows when $\lambda_{\min }\left(W_{o} W_{c}\right)<\frac{1}{4}$.

system $(A, B, C) . \quad T_{\infty}$ is then obtained as the unique symmetric positive definite square root of $P_{\infty}$. In this section we address the general problem of determining all balancing transformations $T \in G L(n, \Re)$ for a given asymptotically stable system $(A, B, C)$, using a suitable gradient flow on the set $G L(n, \Re)$ of all invertible $n \times n$ matrices. This allows us to compute balancing transformations without squaring down an operator; cf. [11]. by

Thus for $T \in G L(n, \Re)$, we consider the cost function $\Phi: G L(n, \Re) \rightarrow \Re$ defined

$$
\Phi(T)=\operatorname{tr}\left(T W_{c} T^{\prime}+\left(T^{\prime}\right)^{-1} W_{o} T^{-1}\right)
$$

and the associated gradient flow $\dot{T}=-\nabla \Phi(T)$ on $G L(n, \Re)$. Of course, to define the gradient of a function, we must specify a Riemannian metric with respect to which the gradient is defined; see the Appendix. Here, as in the previous section, we endow $G L(n, \Re)$ with its standard Riemannian metric

$$
\langle A, B\rangle=2 \operatorname{tr}\left(A^{\prime} B\right),
$$

i.e., with the constant Frobenius inner product (2.12) defined on the tangent spaces of $G L(n, \Re)$.

THEOREM 2.4. Let $W_{c}$ and $W_{o}$ denote the controllability and observability gramians of the asymptotically stable, controllable and observable realization $(A, B, C)$. 
(a) The gradient flow $\dot{T}=-\nabla \Phi(T)$ of $\Phi: G L(n, \Re) \rightarrow \Re$ is

$$
\dot{T}=\left(T^{\prime}\right)^{-1} W_{o}\left(T^{\prime} T\right)^{-1}-T W_{c}
$$

and for any initial condition $T_{0} \in G L(n, \Re)$, the solution $T(t)$ of $(2.13), T(0)=T_{0}$ exists in $G L(n, \Re)$ for all $t \geq 0$.

(b) For any initial condition $T_{0} \in G L(n, \Re)$, the solution $T(t)$ of $(2.13)$ converges to a balancing transformation $T_{\infty} \in G L(n, \Re)$, and all balancing transformations can be obtained in this way for suitable initial conditions $T_{0} \in G L(n, \Re)$.

(c) Let $T_{\infty}$ be a balancing transformation and let $\operatorname{In}\left(T_{\infty}\right)$ denote the set of all $T_{0} \in G L(n, \Re)$, such that the solution $T(t)$ of $(2.13)$ with $T(0)=T_{0}$ converges to $T_{\infty}$ as $t \rightarrow \infty$. Then $\operatorname{In}\left(T_{\infty}\right)$ is an immersed invariant submanifold of $G L(n, \Re)$ of dimension $n(n+1) / 2$ and every solution $T(t) \in \operatorname{In}\left(T_{\infty}\right)$ converges exponentially fast in $\operatorname{In}\left(T_{\infty}\right)$ to $T_{\infty}$.

Proof. $G L(n, \Re)$ is an open subset of $\Re^{n \times n}$ and therefore the tangent space of $G L(n, \Re)$ at $T$ can be identified with the $\Re$-vectorspace of all real $n \times n$ matrices $\xi \in \Re^{n \times n}$. The Fréchet derivative of $\Phi: G L(n, \Re) \rightarrow \Re$, at $T$ is the linear operator on the tangent space of $G L(n, \Re)$ at $T$ defined by

$$
\left.D \Phi\right|_{T}(\xi)=2 \operatorname{tr}\left[\left(W_{c} T^{\prime}-T^{-1}\left(T^{\prime}\right)^{-1} W_{o} T^{-1}\right) \xi\right]=2 \operatorname{tr}\left[\left(T W_{c}-\left(T^{\prime}\right)^{-1} W_{o}\left(T^{\prime} T\right)^{-1}\right)^{\prime} \xi\right]
$$

for all $\xi \in \Re^{n \times n}$. Thus the gradient of $\Phi$ with respect to the Riemannian metric $(2.12)$ is

$$
\nabla \Phi(T)=T W_{c}-\left(T^{\prime}\right)^{-1} W_{o}\left(T^{\prime} T\right)^{-1}
$$

To prove that the gradient flow (2.13) is complete, i.e., that the solutions $T(t)$ exist for all $t \geq 0$, it suffices to show that $\Phi: G L(n, \Re) \rightarrow \Re_{+}$is proper, i.e., that the pre-image $\Phi^{-1}(K)$ of any compact subset $K \subset \Re_{+}$is compact in $G L(n, \Re)$. More generally, a continuous map $f: X \rightarrow Y$ between topological Hausdorff spaces is called proper if the inverse image of $f^{-1}(K)$ of any compact subset $K \subset Y$ is compact. Let $\mathcal{P}(n)=\left\{P \in G L(n, \Re) \mid P=P^{\prime}>0\right\}$. By Lemma 2.1, $P \mapsto \operatorname{tr}\left(W_{c} P+W_{o} P^{-1}\right)$ is a proper function on $\mathcal{P}(n)$. By the polar decomposition, the set of invertible matrices $T$ corresponding to a fixed matrix $T^{\prime} T$ is compact. More generally, we conclude that the map $G L(n, \Re) \rightarrow \mathcal{P}(n), T \mapsto T^{\prime} T$ is proper. Thus $\Phi$ is the composition of proper maps and therefore it is also proper. This shows (a). To prove (b), we note that by (a) and a well-known property of gradient flows, any solution $T(t)$ converges to an equilibrium point $T_{\infty}$ of (2.13).

$$
\left(T_{\infty}^{\prime}\right)^{-1} W_{o}\left(T_{\infty}^{\prime} T_{\infty}\right)^{-1}=T_{\infty} W_{c} \Longleftrightarrow\left(T_{\infty}^{\prime}\right)^{-1} W_{o} T_{\infty}^{-1}=T_{\infty} W_{c} T_{\infty}^{\prime}
$$

and hence $T_{\infty}$ is balancing. This shows (b).

To prove (c), we use the following lemma, where

$$
E:=\left\{T_{\infty} \in G L(n, \Re) \mid\left(T_{\infty}^{\prime} T_{\infty}\right) W_{c}\left(T_{\infty}^{\prime} T_{\infty}\right)=W_{o}\right\}
$$

denotes the set of equilibria points of (2.13).

LEMmA 2.5. The tangent space of $E$ at $T_{\infty} \in E$ is

$$
T_{T_{\infty}} E=\left\{S \in \Re^{n \times n} \mid S^{\prime} T_{\infty}+T_{\infty}^{\prime} S=0\right\} .
$$


Proof. Let $P_{\infty}$ denote the unique symmetric positive definite solution of $P W_{\mathrm{c}} P=$ $W_{\circ}$. Thus $E=\left\{T \mid T^{\prime} T=P_{\infty}\right\}$ and therefore $T_{T_{\infty}} E$ is the kernel of the derivative of $T \mapsto T^{\prime} T-P_{\infty}$ at $T_{\infty}$. Thus $S \in T_{T_{\infty}} E$ if and only if $S^{\prime} T_{\infty}+T_{\infty}^{\prime} S=0$.

Let

$$
\phi(P)=\operatorname{tr}\left(W_{c} P+W_{o} P^{-1}\right)
$$

and

$$
\lambda(T)=T^{\prime} T
$$

Thus $\Phi(T)=\phi(\lambda(T))$. By Theorem 2.2, see also [4] and [2],

(i) $\left.D \phi\right|_{P_{\infty}}=0$.

(ii) $\left.D^{2} \phi\right|_{P_{\infty}}>0$.

Let $X$ denote the matrix representing the linear operator $\left.D \lambda\right|_{T_{\infty}}(S)=T_{\infty}^{\prime} S+$ $S^{\prime} T_{\infty}$. Using the chain rule, we obtain

$$
\left.D^{2} \Phi\right|_{T_{\infty}}=\left.X^{\prime} \cdot D^{2} \phi\right|_{P_{\infty}} \cdot X
$$

for all $T_{\infty} \in E$. By (ii) and (2.16), $\left.D^{2} \Phi\right|_{T_{\infty}} \geq 0$ and $D^{2} \Phi \mid T_{\infty}$ degenerates exactly on the kernel of $X$, i.e., on the tangent space $T_{T_{\infty}} E$. Thus $\Phi$ is a Morse-Bott function; see the Appendix. Thus Proposition A.3 implies that every solution $T(t)$ of (2.13) converges to an equilibrium point. Moreover, the equilibrium set $E$ is normally hyperbolic.

It follows from the theory of stable manifolds (see, e.g., Irwin [12]) that $\operatorname{In}\left(\mathcal{T}_{\infty}\right)$ is the stable manifold of (2.13) at $T_{\infty}$ and thus is an immersed invariant submanifold of $G L(n, \Re)$ of $\operatorname{dimension} \operatorname{dim} G L(n, \Re)-\operatorname{dim} E=n^{2}-n(n-1) / 2=n(n+1) / 2$. Since the convergence is always exponential on stable manifolds, this completes the proof of (c).

Now consider the following quadratic version of our objective function $\Phi$. For $T \in G L(n, \Re)$, let $\Psi: G L(n, \Re) \rightarrow \Re$ be defined by

$$
\Psi(T):=\operatorname{tr}\left(\left(T W_{c} T^{\prime}\right)^{2}+\left(\left(T^{\prime}\right)^{-1} W_{o} T^{-1}\right)^{2}\right) .
$$

The gradient flow $\dot{T}=-\nabla \Psi(T)$ on $G L(n, \Re)$ is easily computed to be

$$
\dot{T}=\left(T^{\prime}\right)^{-1} W_{o}\left(T^{\prime} T\right)^{-1} W_{o}\left(T^{\prime} T\right)^{-1}-T W_{c} T^{\prime} T W_{c} .
$$

The same arguments as for Theorem 2.4 show that for all initial conditions $T_{o} \in$ $G L(n, \Re)$, the solution $T(t) \in G L(n, \Re)$ of $(2.18)$ exists for all $t \geq 0$ and converges to a balancing transformation for $(A, B, C)$. Thus we can also use (2.18) or suitable discretized versions to compute balancing transformations for a given asymptotically stable minimal realization $(A, B, C)$. We illustrate the behaviour of the gradient flows (2.13) and (2.18) by means of the following simulation experiments.

In Fig. 2 the diagonal entries of $T(t)$ are plotted. Figure $2(\mathrm{a})$ uses $W_{o}=W_{1}$, $W_{c}=W_{2}$ and a starting value of $T_{0}=P_{1}$, in (2.13). Figure 2(b) has the same value for the gramians, but has a starting value of

$$
T_{0}=\left[\begin{array}{llll}
1 & 3 & 4 & 2 \\
4 & 3 & 2 & 5 \\
3 & 2 & 4 & 1 \\
2 & 4 & 3 & 4
\end{array}\right]
$$




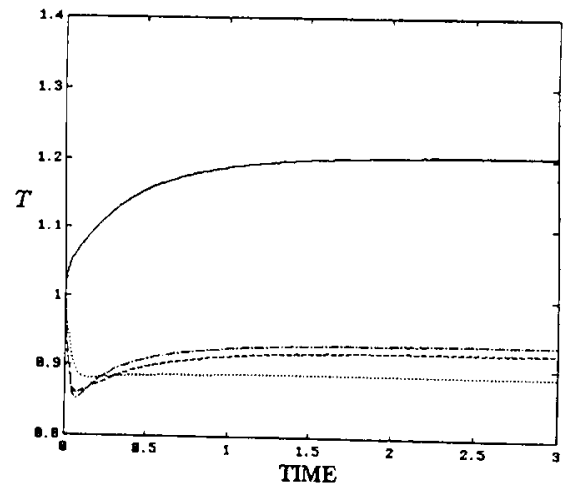

(a)

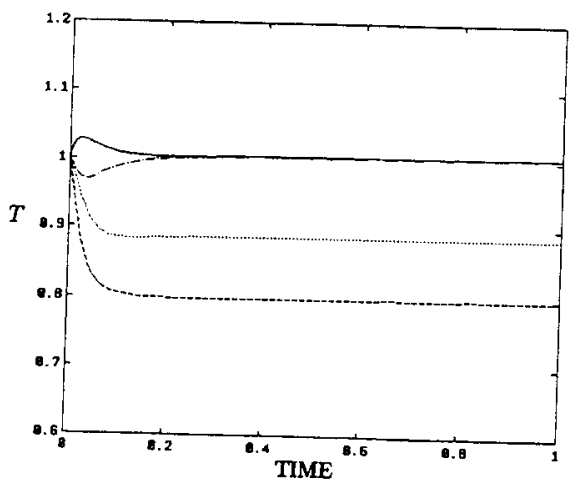

(c)

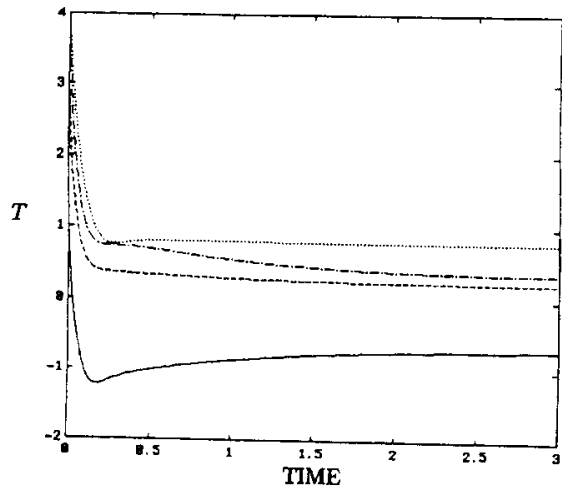

(b)

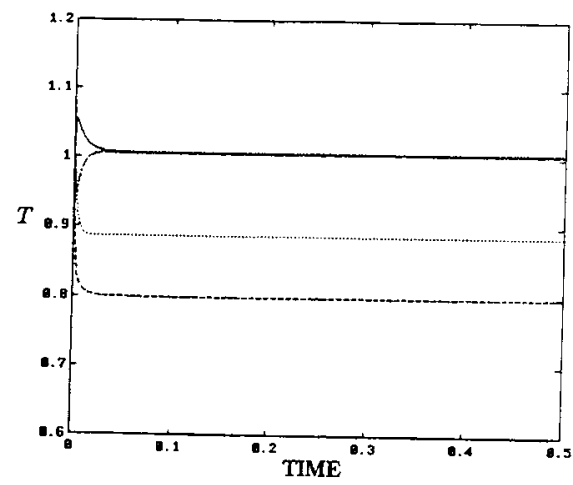

(d)

FIG. 2. Comparison of linear and quadratic index flows on $G L(n, \Re)$. (a) Linear flow on $T$ when $\lambda_{\min }\left(W_{o} W_{c}\right)<\frac{1}{4}$. (b) Quadratic flow on $T$ when $\lambda_{\min }\left(W_{o} W_{c}\right)<\frac{1}{4}$. (c) Linear flow on $T$ when $\lambda_{\min }\left(W_{o} W_{c}\right)>\frac{1}{4}$. (d) Quadratic flow on $T$ when $\lambda_{\min }\left(W_{o} W_{c}\right)>\frac{1}{4}$.

It can be observed that these $T_{0}$ values give different final solutions, both of which are generalized balancing transformations. Figures 2(c)-(d) use $W_{0}=W_{3}, W_{c}=W_{4}$ and a starting value of $T_{0}=P_{1}$. Figure 2(c) uses (2.13) while Fig. 2(d) uses (2.18). Note that in this case, (2.18) converges more rapidly than (2.13).

2.3. Diagonal balancing transformations. Here we address the related issue of computing diagonal balancing transformations $T$ for a given asymptotically stable
minimal realization, i.e., $T$ satisfies

$$
T W_{c} T^{\prime}=\left(T^{\prime}\right)^{-1} W_{o} T^{-1}=\text { diagonal. }
$$

Any such diagonal balancing transformation $T$ is of the form $T=\Theta \cdot T_{\infty}$, where $T_{\infty}=P_{\infty}^{1 / 2}$ is the uniquely determined positive definite symmetric balancing transformation whose existence is guaranteed by Theorem 2.1 and where $\Theta$ is an orthogonal matrix that diagonalizes $T_{\infty} W_{c} T_{\infty}^{\prime}=\left(T_{\infty}^{\prime}\right)^{-1} W_{o} T_{\infty}^{-1}$.

Let us consider a fixed diagonal positive definite matrix $N=\operatorname{diag}\left(\lambda_{1}, \ldots, \lambda_{n}\right)$ with distinct eigenvalues $\lambda_{1}>\cdots>\lambda_{n}>0$. Using $N$, a weighted cost function for balancing is defined by

$$
\Phi_{N}(T)=\operatorname{tr}\left(N T W_{c} T^{\prime}+N\left(T^{\prime}\right)^{-1} W_{o} T^{-1}\right) .
$$


The following lemma characterizes the diagonal balancing transformations as the critical points of the weighted cost function $\Phi_{N}$ on $G L(n, \Re)$.

LEMma 2.6. Let $N=\operatorname{diag}\left(\lambda_{1}, \ldots, \lambda_{n}\right)$ with $\lambda_{1}>\cdots>\lambda_{n}>0$ and let $W_{c}, W_{o}$ denote the controllability and observability gramians of an asymptotically stable minimal realization $(A, B, C)$. Then

(a) $T \in G L(n, \Re)$ is a critical point of $\Phi_{N}: G L(n, \Re) \rightarrow \Re, \Phi_{N}(T)=\operatorname{tr}\left(N T W_{c} T^{\prime}+\right.$ $\left.N\left(T^{\prime}\right)^{-1} W_{o} T^{-1}\right)$, if and only if $T$ is a diagonal balancing transformation, i.e.,

$$
T W_{c} T^{\prime}=\left(T^{\prime}\right)^{-1} W_{o} T^{-1}=\text { diagonal. }
$$

(b) $\Phi_{N}: G L(n, \Re) \rightarrow \Re$ has compact sublevel sets. In particular, a global minimum $T_{\min } \in G L(n, \Re)$ of $\Phi_{N}: G L(n, \Re) \rightarrow \Re$ exists.

Proof. The Fréchet derivative of $\Phi_{N}: G L(n, \Re) \rightarrow \Re$ at $T$ is the linear map defined by

$$
\begin{aligned}
\left.D \Phi_{N}\right|_{T}(\xi) & =2 \operatorname{tr}\left(N \xi W_{c} T^{\prime}-N\left(T^{\prime}\right)^{-1} W_{o} T^{-1} \xi T^{-1}\right) \\
& =2 \operatorname{tr}\left[\left(N T W_{c}-\left(T^{\prime}\right)^{-1} W_{o} T^{-1} N\left(T^{\prime}\right)^{-1}\right)^{\prime} \xi\right]
\end{aligned}
$$

and therefore the gradient of $\Phi_{N}(T)$ with respect to the Riemannian metric (2.12) on $G L(n, \Re)$ is

$$
\nabla \Phi_{N}(T)=N T W_{c}-\left(T^{\prime}\right)^{-1} W_{o} T^{-1} N\left(T^{\prime}\right)^{-1}
$$

It follows that $T \in G L(n, \Re)$ is a critical point of $\Phi_{N}$ if and only if $\nabla_{N}(T)=0$, i.e., if and only if

$$
N T W_{c} T^{\prime}=\left(T^{\prime}\right)^{-1} W_{o} T^{-1} \cdot N
$$

By symmetry of $T W_{c} T^{\prime}$ and $\left(T^{\prime}\right)^{-1} W_{o} T^{-1}$, we obtain from (2.22) that

$$
\begin{aligned}
N^{2} T W_{c} T^{\prime} & =T W_{c} T^{\prime} N^{2} \\
N^{2}\left(T^{\prime}\right)^{-1} W_{o} T^{-1} & =\left(T^{\prime}\right)^{-1} W_{o} T^{-1} N^{2} .
\end{aligned}
$$

Any symmetric matrix that commutes with $N^{2}$ must be diagonal, since $N^{2}$ has distinct eigenvalues. Thus we see that $(2.22)$ is equivalent to $T W_{c} T^{\prime}=\left(T^{\prime}\right)^{-1} W_{o} T^{-1}=$ diagonal. This proves (a). For (b) note that $\Phi_{N}(T) \leq a$ implies

$$
\left\|N^{1 / 2} T W_{c}^{1 / 2}\right\|^{2} \leq a, \quad\left\|W_{o}^{1 / 2} T^{-1} N^{1 / 2}\right\|^{2} \leq a
$$

for the Frobenius norm $\|X\|^{2}=\operatorname{tr}\left(X X^{\prime}\right)$. Hence $\|T\| \leq c_{1},\left\|T^{-1}\right\| \leq c_{2}$ for positive constants $c_{1}, c_{2}$ that depend only on $N, W_{c}, W_{\circ}$ and $a$. Thus $\left\{T \in G L(n, \Re) \mid \Phi_{N}(T) \leq\right.$ $a\}$ is a closed subset of the compact set $\left\{T \in G L(n, \Re) \mid\|T\| \leq c_{1},\left\|T^{-1}\right\| \leq c_{2}\right\}$ and therefore also compact. This shows that $\Phi_{N}: G L(n, \Re) \rightarrow \Re$ has compact sublevel sets. But any continuous function $f: G L(n, \Re) \rightarrow \Re_{+}$with compact sublevel sets has a minimizing $T \in G L(n, \Re)$. This completes the proof of Lemma 2.6 .

From Lemma 2.6 and by (2.20), similar arguments as for Theorem 2.4(a) and (b) show the following theorem.

THEOREM 2.7. Let $W_{c}, W_{o}$ be the controllability and observability gramians of the asymptotically stable, controllable, and observable realization $(A, B, C)$ and let $N=\operatorname{diag}\left(\lambda_{1}, \ldots, \lambda_{n}\right)$ with $\lambda_{1}>\cdots>\lambda_{n}>0$. Then 
(a) gradient flow $\dot{T}=-\nabla \Phi_{N}(T)$ of the weighted cost function $\Phi_{N}: G L(n, \Re) \rightarrow$ $\Re$ is

$$
\dot{T}=\left(T^{\prime}\right)^{-1} W_{o} T^{-1} N\left(T^{\prime}\right)^{-1}-N T W_{c}
$$

For all initial conditions $T(0) \in G L(n, \Re)$, the solution $T(t) \in G L(n, \Re)$ of $(2.24)$ exists for all $t \geq 0$.

(b) For any initial condition $T(0) \in G L(n, \Re)$, the solution $T(t)$ of $(2.24)$ converges to a diagonal balancing transformation $T_{\infty}$ of $(A, B, C)$.

(c) Suppose that the singular values $0<d_{1}<\cdots<d_{n}$ of the Hankel operator of $(A, B, C)$ are distinct. Then the stable equilibrium of (2.24) are characterized by $\left(T_{\infty}^{\prime}\right)^{-1} W_{o} T_{\infty}^{-1}=T_{\infty} W_{c} T_{\infty}^{\prime}=D$, where $D=\operatorname{diag}\left(d_{1}, \ldots, d_{n}\right)$ is diagonal and the diagonal entries are in reverse ordering to those of $N$. Moreover, the gradient flow (2.24) converges exponentially fast to the $2^{n}$ stable equilibria with a convergence rate lower bounded by

$$
\lambda_{\min }\left(\left(T_{\infty} T_{\infty}^{\prime}\right)^{-1}\right) \min _{i<j}\left[\left(d_{i}-d_{j}\right)\left(\lambda_{j}-\lambda_{i}\right), 4 d_{i} \lambda_{i}\right]
$$

All other equilibria are unstable.

Proof. Parts (a) and (b) follow easily from Lemma 2.6, using similar arguments as for Theorem 2.4. To prove (c), consider the linearization of (2.24) at an equilibrium point $T_{\infty}$; that is, where $\left(T_{\infty}^{\prime}\right)^{-1} W_{o} T_{\infty}^{-1}=T_{\infty} W_{c} T_{\infty}^{\prime}=D$ and

$$
\begin{aligned}
\dot{\eta}= & -N \eta T_{\infty}^{-1} D\left(T_{\infty}^{\prime}\right)^{-1}-D \eta T_{\infty}^{-1} N\left(T_{\infty}^{\prime}\right)^{-1} \\
& -\left(T_{\infty}^{\prime}\right)^{-1} \eta^{\prime} D N\left(T_{\infty}^{\prime}\right)^{-1}-D N\left(T_{\infty}^{\prime}\right)^{-1} \eta^{\prime}\left(T_{\infty}^{\prime}\right)^{-1} .
\end{aligned}
$$

Let $\zeta=\eta T_{\infty}^{-1}$, then

$$
\dot{\zeta}\left(T_{\infty} T_{\infty}^{\prime}\right)=-N \zeta D-D \zeta N-\zeta^{\prime} D N-D N \zeta^{\prime}
$$

and thus, using Kronecker products and the vec notation, and recalling that $\operatorname{vec}(A B C)$ $=\left(C^{\prime} \otimes A\right) \operatorname{vec}(B)$, then

$$
\left[\left(T_{\infty} T_{\infty}^{\prime}\right) \otimes I\right] \operatorname{vec}(\dot{\zeta})=-[D \otimes N+N \otimes D] \operatorname{vec}(\zeta)-[D N \otimes I+I \otimes D N] \operatorname{vec}\left(\zeta^{\prime}\right)
$$

Consider first the special case when $T_{\infty} T_{\infty}^{\prime}=I$, and $\zeta$ is denoted $\zeta^{*}$ :

$$
\operatorname{vec}\left(\dot{\zeta}^{*}\right)=-[D \otimes N+N \otimes D] \operatorname{vec}\left(\zeta^{*}\right)-[D N \otimes I+I \otimes D N] \operatorname{vec}\left(\zeta^{* \prime}\right)
$$

Then for $i<j$,

$$
\left[\begin{array}{c}
\dot{\zeta}_{i j}^{*} \\
\dot{\zeta}_{j i}^{*}
\end{array}\right]=-\left[\begin{array}{cc}
d_{i} \lambda_{j}+\lambda_{i} d_{j} & d_{j} \lambda_{j}+d_{i} \lambda_{i} \\
d_{i} \lambda_{i}+d_{j} \lambda_{j} & d_{i} \lambda_{j}+\lambda_{i} d_{j}
\end{array}\right]\left[\begin{array}{c}
\zeta_{i j}^{*} \\
\zeta_{j i}^{*}
\end{array}\right]
$$

and for all $i$,

$$
\dot{\zeta}_{i i}^{*}=-4 d_{i} \lambda_{i} \zeta_{i i}^{*}
$$

By assumption, $\lambda_{i}>0$, and $d_{i}>0$ for all $i$. Thus (2.25) is exponentially stable if and only if $\left(d_{i}-d_{j}\right)\left(\lambda_{j}-\lambda_{i}\right)>0$ for all $i, j, i<j$, or equivalently, if and only if the diagonal entries of $D$ are distinct and in reverse ordering to those of $N$. In this case, $(2.25)$ is equivalent to $(2.26)$

$$
\operatorname{vec}\left(\dot{\zeta}^{*}\right)=-\mathcal{F} \operatorname{vec}\left(\zeta^{*}\right)
$$




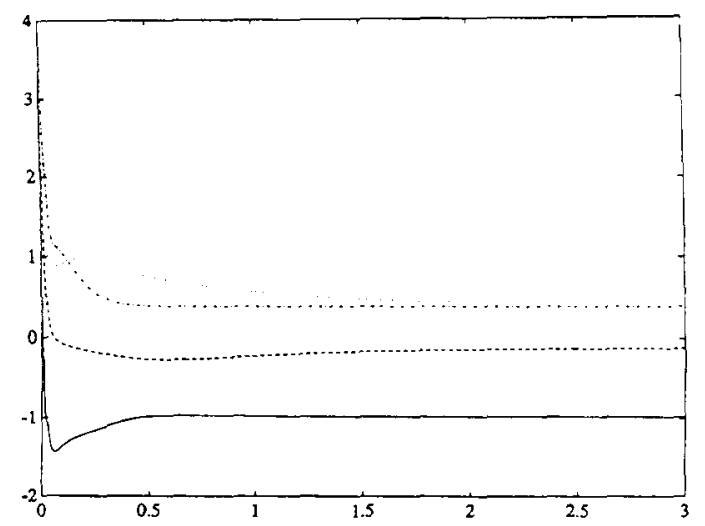

FIG. 3. Evolution of the diagonalizing transformation $T$.

with a symmetric positive definite matrix $\mathcal{F}=\mathcal{F}^{\prime}>0$.

Consequently, there is exponential convergence with a rate given by $\lambda_{\min }(\mathcal{F})$ as follows:

$$
\begin{aligned}
\lambda_{\min }(\mathcal{F}) & =\min \left(\min _{i<j}\left[\lambda_{\min }\left(\left[\begin{array}{cc}
d_{i} \lambda_{j}+\lambda_{i} d_{j} & d_{j} \lambda_{j}+d_{i} \lambda_{i} \\
d_{i} \lambda_{i}+d_{j} \lambda_{j} & d_{i} \lambda_{j}+\lambda_{i} d_{j}
\end{array}\right]\right)\right], \min _{i}\left[4 d_{i} \lambda_{i}\right]\right) \\
& =\min \left(\min _{i<j}\left[d_{i} \lambda_{j}+\lambda_{i} d_{j}-d_{j} \lambda_{j}-d_{i} \lambda_{i}\right], \min _{i}\left[4 d_{i} \lambda_{i}\right]\right) \\
& =\min \left(\min _{i<j}\left[\left(d_{i}-d_{j}\right)\left(\lambda_{j}-\lambda_{i}\right)\right], \min _{i}\left[4 d_{i} \lambda_{i}\right]\right) .
\end{aligned}
$$

Relaxing the assumption $T_{\infty} T_{\infty}^{\prime}=I$ is possible since $T_{\infty} T_{\infty}^{\prime}$ is positive definite so that $\left(T_{\infty} T_{\infty}^{\prime} \otimes I\right)$ is positive definite. Thus exponential stability of $(2.26)$ assures exponential stability of

$$
\left[\left(T_{\infty} T_{\infty}^{\prime}\right) \otimes I\right] \operatorname{vec}(\dot{\zeta})=-\mathcal{F}_{\operatorname{vec}(\zeta)}
$$

The rate of exponential convergence is given by $\lambda_{\min }\left[\left(\left(T_{\infty} T_{\infty}^{\prime}\right)^{-1} \otimes I\right) \mathcal{F}\right]$. Now since $A=A^{\prime}>0, B=B^{\prime}>0$ implies $\lambda_{\min }(A B) \geq \lambda_{\min }(A) \lambda_{\min }(B)$, a lower bound on the convergence rate is given from

$$
\begin{aligned}
& \lambda_{\min }\left[\left(\left(T_{\infty} T_{\infty}^{\prime}\right)^{-1} \otimes I\right) \mathcal{F}\right] \geq \lambda_{\min }\left[\left(T_{\infty} T_{\infty}^{\prime}\right)^{-1} \otimes I\right] \lambda_{\min }(\mathcal{F}) \\
& =\lambda_{\min }\left[\left(T_{\infty} T_{\infty}^{\prime}\right)^{-1}\right] \min \left(\min _{i<j}\left[\left(d_{i}-d_{j}\right)\left(\lambda_{j}-\lambda_{i}\right)\right], \min _{i}\left[4 d_{i} \lambda_{i}\right]\right)
\end{aligned}
$$

as claimed.

Simulation. In Fig. 3 the diagonal elements of $T(t)$ are plotted. The flow (2.24) is allowed to evolve with $W_{o}=W_{1}, W_{c}=W_{2}, N=\operatorname{diag}(5,4,3,2)$, and initial matrix $T_{0}$ as before. At $t=3$,

$$
T(t)=\left[\begin{array}{cccc}
-0.9788 & 0.6595 & -0.1033 & 0.6623 \\
-0.0807 & -0.1124 & -0.7002 & 0.5847 \\
0.1079 & -0.4691 & 0.4256 & 0.1943 \\
0.7586 & 0.5177 & 0.4245 & 0.3909
\end{array}\right]
$$


and this transformation gives

$$
\begin{aligned}
W_{\mathrm{c}} & =\left[\begin{array}{cccc}
0.4554 & 0.0017 & 0.0006 & -0.0005 \\
0.0017 & 2.7493 & 0.0034 & -0.0000 \\
0.0006 & 0.0034 & 3.3641 & -0.0034 \\
-0.0005 & -0.0000 & -0.0034 & 11.3114
\end{array}\right], \\
W_{o} & =\left[\begin{array}{cccc}
0.4553 & 0.0021 & 0.0009 & 0.0014 \\
0.0021 & 2.7496 & 0.0042 & 0.0007 \\
0.0009 & 0.0042 & 3.3625 & 0.0081 \\
0.0014 & 0.0007 & 0.0081 & 11.3090
\end{array}\right] .
\end{aligned}
$$

Notice that although convergence has not been completed, the gramians are diagonally dominant with increasing elements.

3. Differential equations for balanced realizations. In this section we construct certain ordinary differential equations

$$
\begin{aligned}
& \dot{A}=f(A, B, C) \\
& \dot{B}=g(A, B, C) \\
& \dot{C}=h(A, B, C)
\end{aligned}
$$

evolving on the space of all realizations (A,B,C) of a given transfer function $G(s)$, with the property that their solutions $(A(t), B(t), C(t))$ all converge for $t \rightarrow \infty$ to balanced realizations $(\bar{A}, \bar{B}, \bar{C})$ of $G(s)$.

Let $G(s) \in \Re(s)^{p \times m}$ denote an asymptotically stable strictly proper real rational transfer function of McMillan degree $n$. Thus $G(s)$ has its poles either in the open left half-plane or in the open unit disc, respectively. We denote by $(A, B, C) \in$ $\Re^{n \times(n+m+p)}$ an asymptotically stable, controllable, and observable realization of $G(s)$, i.e., $G(s)=C(s I-A)^{-1} B$.

Let

$$
\mathcal{R}_{G}=\left\{(A, B, C) \in \Re^{n \times(n+m+p)} \mid G(s)=C(s I-A)^{-1} B\right\}
$$

denote the set of all minimal state space realizations of the transfer function $G(s)$. By Kalman's realization theorem, [8]

$$
\mathcal{R}_{G}=\left\{\left(T A T^{-1}, T B, C T^{-1}\right) \in \Re^{n \times(n+m+p)} \mid T \in G L(n, \Re)\right\}
$$

for any fixed initial realization $(A, B, C) \in \mathcal{R}_{G}$. Thus $\mathcal{R}_{G}$ is an orbit of the $G L(n, \Re)$ similarity action $(A, B, C) \mapsto\left(T A T^{-1}, T B, C T^{-1}\right)$ on $\Re^{n \times(n+m+p)}$.

We consider the function

$$
\Phi: \mathcal{R}_{G} \rightarrow \Re
$$

defined by

$$
\Phi(A, B, C)=\operatorname{tr}\left(W_{c}(A, B)+W_{o}(A, C)\right),
$$

i.e., by the sum of the eigenvalues of the controllability and observability gramians of $(A, B, C)$. The following proposition summarizes some important properties of $\mathcal{R}_{G}$ and $\Phi: \mathcal{R}_{G} \rightarrow \Re$.

Proposition 3.1. It holds that 
(a) $\mathcal{R}_{G}$ is a smooth, closed submanifold of $\Re^{n \times(n+m+p)}$. The tangent space of $\mathcal{R}_{G}$ at $(A, B, C) \in \mathcal{R}_{G}$ is

$$
T_{(A, B, C)} \mathcal{R}_{G}=\left\{(X A-A X, X B,-C X) \mid X \in \Re^{n \times n}\right\} .
$$

(b) The function $\Phi: \mathcal{R}_{G} \rightarrow \Re$ defined by (3.3) is smooth and has compact sublevel sets.

Proof. $\mathcal{R}_{G}$ is an orbit of the $G L(n, \Re)$-similarity action

$$
\begin{aligned}
\sigma: G L(n, \Re) \times \Re^{n \times(n+m+p)} & \rightarrow \Re^{n \times(n+m+p)} \\
(T,(A, B, C)) & \mapsto\left(T A T^{-1}, T B, C T^{-1}\right)
\end{aligned}
$$

and thus, by a general result about algebraic Lie group actions (see, e.g., Appendix $\mathrm{C}$ in [9]) is a smooth submanifold of the Euclidean space $\Re^{n \times(n+m+p)}$. By Lemma $3.3[2], \mathcal{R}_{G}$ is a closed subset of $\Re^{n(n+m+p)}$ if $(A, B, C)$ is controllable and observable. Explicitly, by realization theory, $\mathcal{R}_{G}$ is a fiber of the continuous map

$$
\begin{aligned}
f: \Re^{n(n+m+p)} & \rightarrow \prod_{i=0}^{\infty} \Re^{p \times m} \\
(F, G, H) & \mapsto\left(H F^{i} G \mid i \in N N_{o}\right)
\end{aligned}
$$

and therefore closed.

To prove (b) and (3.4), we consider the diffeomorphism

$$
\begin{aligned}
\sigma: G L(n, \Re) & \rightarrow \mathcal{R}_{G} \\
T & \mapsto\left(T A T^{-1}, T B, C T^{-1}\right)
\end{aligned}
$$

(this requires that $(A, B, C)$ is minimal). The derivative of $\sigma$ at the identity matrix is the linear map $X \mapsto(X A-A X, X B,-C X)$, which maps $\Re^{n \times n}$ onto $T_{(A, B, C)} \mathcal{R}_{G}$. This proves (3.4). Furthermore, with $P=T^{\prime} T$,

$$
\begin{aligned}
\Phi(\sigma(T)) & =\operatorname{tr}\left(T W_{c}(A, B) T^{\prime}+\left(T^{\prime}\right)^{-1} W_{o}(A, C) T^{-1}\right) \\
& =\operatorname{tr}\left(W_{c} P+W_{o} P^{-1}\right),
\end{aligned}
$$

and the result now follows from Lemma 2.1 , i.e., that the function $P \mapsto \operatorname{tr}\left(W_{c} P+\right.$ $\left.W_{\circ} P^{-1}\right)$ on the set of positive definite symmetric matrices has compact sublevel sets.

We now address the issue of finding gradient flows for the objective function $\Phi: \mathcal{R}_{G} \rightarrow \Re$ relative to some Riemannian metric on $\mathcal{R}_{G}$. While there are several possible choices for a Riemannian metric on the realization space $\mathcal{R}_{G}$, the following one leads to a particularly simple expression for the gradient.

In the sequel, we use the Lie bracket notation

$$
[A, B]=A B-B A
$$

for $n \times n$ matrices $A, B$.

Given two tangent vectors $\left(\left[X_{1}, A\right], X_{1} B,-C X_{1}\right)$ and $\left(\left[X_{2}, A\right], X_{2} B,-C X_{2}\right) \in$ $T_{(A, B, C)} \mathcal{R}_{G}$ we define

$$
\left\langle\left\langle\left(\left[X_{1}, A\right], X_{1} B,-C X_{1}\right), \quad\left(\left[X_{2}, A\right], X_{2} B,-C X_{2}\right)\right\rangle\right\rangle:=\operatorname{tr}\left(X_{1}^{\prime} X_{2}\right) .
$$


To prove that (3.8) defines an inner product on $T_{(A, B, C)} \mathcal{R}_{G}$, we need the following lemma.

LEMMA 3.2. Let $(A, B, C)$ be controllable or observable. Then $([X, A], X B,-C X)$ $=(0,0,0)$ implies $X=0$.

Proof. If $X B=0$ and $A X=X A$, then $X\left(B, A B, \ldots, A^{n-1} B\right)=0$. Thus controllability implies $X=0$. This is also true for observability.

It is now easily seen, using Lemma 3.2 , that (3.8) defines a nondegenerate symmetric bilinear form on each tangent space $T_{(A, B, C)} \mathcal{R}_{G}$ and in fact a Riemannian metric on $\mathcal{R}_{G}$. We refer to this as the normal Riemannian metric on $\mathcal{R}_{G}$.

To determine the gradient flow of $\Phi: \mathcal{R}_{G} \rightarrow \Re$ with respect to the normal Riemannian metric, we need a lemma.

LEMMA 3.3. Let $N \in \Re^{n \times n}$ be a real symmetric $n \times n$ matrix and let $\Phi_{N}: \mathcal{R}_{G} \rightarrow$ $\Re$ be defined by $\Phi_{N}(A, B, C)=\operatorname{tr}\left(N W_{c}(A, B)+N W_{o}(A, C)\right)$ for all $(A, B, C) \in \mathcal{R}_{G}$. Then the Fréchet derivative of $\Phi_{N}$ at $(A, B, C) \in \mathcal{R}_{G}$ is the linear map $D \Phi_{N}(A, B, C)$ : $T_{(A, B, C)} \mathcal{R}_{G} \rightarrow \Re$ defined by

$$
D \Phi_{N}(A, B, C)([X, A], X B,-C X)=2 \operatorname{tr}\left[\left(W_{c}(A, B) N-N W_{o}(A, C)\right) X\right]
$$

for $X \in \Re^{n \times n}$.

Proof. Let $\sigma: G L(n, \Re) \rightarrow \mathcal{R}_{G}$ be the diffeomorphism defined by $\sigma(T)=$ $\left(T A T^{-1}, T B, C T^{-1}\right)$. The derivative of $\sigma$ at the identity matrix $I_{n}$ is the linear map $X \mapsto([X, A], X B,-C X)$ on $\Re^{n \times n}$. By the chain rule for the composed map $\Phi_{N} \circ \sigma$ defined by

$$
\Phi_{N}(\sigma(T))=\operatorname{tr}\left(N T W_{c}(A, B) T^{\prime}+N\left(T^{\prime}\right)^{-1} W_{o}(A, C) T^{-1}\right)
$$

we have

$$
\begin{aligned}
D \Phi_{N}\left(\sigma\left(I_{n}\right)\right)([X, A],-X B, C X) & =D\left(\Phi_{N} \circ \sigma\right)\left(I_{n}\right) X \\
& =2 \operatorname{tr}\left(N X W_{c}(A, B)-N W_{o}(A, C) X\right) \\
& =2 \operatorname{tr}\left[\left(W_{c}(A, B) N-N W_{o}(A, C)\right) X\right]
\end{aligned}
$$

for all $X \in \Re^{n \times n}$. The result follows.

THEOREM 3.4. Let $\Phi: \mathcal{R}_{G} \rightarrow \Re$ be the objective function defined by $\Phi(A, B, C)=$ $\frac{1}{2} \operatorname{tr}\left(W_{c}(A, B)+W_{o}(A, C)\right)$.

(a) The gradient flow $\left(\dot{A}=-\operatorname{grad}_{A} \Phi(A, B, C), \dot{B}=-\operatorname{grad}_{B} \Phi(A, B, C), \dot{C}=\right.$ $\left.-\operatorname{grad}_{A} \Phi(A, B, C)\right)$ of $\Phi$ for the normal Riemannian metric on $\mathcal{R}_{G}$ is

$$
\begin{aligned}
\dot{A} & =\left[A, W_{o}(A, C)-W_{c}(A, B)\right] \\
\dot{B} & =\left(W_{o}(A, C)-W_{c}(A, B)\right) B \\
\dot{C} & =C\left(W_{c}(A, B)-W_{o}(A, C)\right) .
\end{aligned}
$$

For every initial condition $(A(0), B(0), C(0)) \in \mathcal{R}_{G}$, the solution $(A(t), B(t), C(t)) \in$ $\mathcal{R}_{G}$ of (3.10) exists for all $t \geq 0$ and converges for $t \rightarrow+\infty$ to a balanced realization $(\bar{A}, \bar{B}, \bar{C})$ of $G(s)$ :

$$
W_{c}(\bar{A}, \vec{B})=W_{o}(\bar{A}, \vec{C})
$$

(b) Convergence to the class of balanced realizations is exponentially fast.

(c) The transfer function of any solution $(A(t), B(t), C(t))$ of $(3.10)$ is independent of $t$. 
Proof. By definition of a gradient,

$$
\operatorname{grad} \Phi(A, B, C)=\left(\operatorname{grad}_{A} \Phi(A, B, C), \operatorname{grad}_{B} \Phi(A, B, C), \operatorname{grad}_{C} \Phi(A, B, C)\right)
$$

is characterized by the properties (see the Appendix)

$$
\operatorname{grad} \Phi(A, B, C) \in T_{(A, B, C)} \mathcal{R}_{G},
$$

and

(3.11b) $D \Phi(A, B, C)([X, A], X B,-C X)=\langle\langle\operatorname{grad} \Phi(A, B, C),([X, A], X B,-C X)\rangle\rangle$

for all $X \in \Re^{n \times n}$. By Proposition 3.1 and Lemma 3.2,

$$
\operatorname{grad} \Phi(A, B, C)=([A, \Lambda], \Lambda B,-C \Lambda)
$$

for a uniquely determined $\Lambda \in \Re^{n \times n}$. Applying Lemma 3.3 for $N=\frac{1}{2} I_{n}$, we see that $(3.11 \mathrm{~b})$ is equivalent to

$$
\begin{aligned}
\operatorname{tr}\left[\left(W_{c}(A, B)-W_{o}(A, C)\right) X\right] & =\langle\langle([A, \Lambda], \Lambda B,-C \Lambda),([A, X], X B,-C X)\rangle\rangle \\
& =\operatorname{tr}\left(\Lambda^{\prime} X\right)
\end{aligned}
$$

for all $X \in \Re^{n \times n}$. Thus

$$
\Lambda=W_{c}(A, B)-W_{o}(A, C)
$$

and $\operatorname{grad} \Phi(A, B, C)=([A, \Lambda], \Lambda B,-C \Lambda)$. This proves (3.10). Since (3.10) is minus the gradient flow of $\Phi, \Phi(A(t), B(t), C(t))$ decreases on any solution of (3.10). By Proposition 3.1(b), $\left\{(A, B, C) \in \mathcal{R}_{G} \mid \Phi(A, B, C) \leq \Phi(A(0), B(0), C(0))\right\}$ is a compact subset of $\mathcal{R}_{G}$, which is invariant under the flow of $(3.10)$. Therefore $(A(t), B(t), C(t))$ stays in that compact subset and thus exists for all $t \geq 0$. The equilibria of (3.10) are characterized by $W_{c}(A, B)=W_{o}(A, C)$, i.e., by the balanced realizations. This proves (a), except that we have not yet established convergence to an equilibrium point.

To prove (b), we consider the diffeomorphism $\sigma: G L(n, \Re) \rightarrow \mathcal{R}_{G}$ defined by $\sigma(T)=\left(T A T^{-1}, T B, C T^{-1}\right)$ for any $(A, B, C) \in \mathcal{R}_{G}$. At each critical point, $(A, B, C)$ of $\Phi: \mathcal{R}_{G} \rightarrow \Re, \sigma$ induces an invertible congruence transformation between the Hessian of $\Phi: \mathcal{R}_{G} \rightarrow \Re$ at $(A, B, C)$ and the Hessian of $\Phi \circ \sigma$ at $I_{n}$. By (2.16) and the proof of Theorem 2.4(c), the Hessian of $\Phi \circ \sigma$ at $I_{n}$ is positive semidefinite and degenerates exactly on the tangent space (at $I_{n}$ ) of the set of balancing transformations. Therefore the Hessian of $\Phi$ at a balanced realization $(A, B, C)$ is positive semidefinite and degenerates exactly on the tangent space of the set of balanced realizations at $(A, B, C)$. (N.B. By Lemma 2.5, the set of balanced realizations of $G(s)$ can be shown to be a smooth submanifold of $\mathcal{R}_{G}$.) This proves (b). As $\Phi: \mathcal{R}_{G} \rightarrow \Re$ is now seen as a Morse-Bott function, we can apply Proposition A.3 to conclude that $(A(t), B(t), C(t))$ converges to an equilibrium point.

Part (c) is obvious, as the flow evolves on $\mathcal{R}_{G}$.

We emphasize that Theorem 3.4 gives, for the first time, a direct method to compute balanced realizations, without computing any balancing transformations. We regard this as one of the really new insights that can be obtained by our ODE methods.

Remark. As is shown in the above proof, any flow on symmetric matrices

$$
\begin{aligned}
& \dot{A}=-[A, \Lambda(A, B, C)] \\
& \dot{B}=-\Lambda(A, B, C) B \\
& \dot{C}=+C \Lambda(A, B, C),
\end{aligned}
$$


where $\Lambda(A, B, C) \in \Re^{n \times n}$ is an arbitrary matrix valued function of $(A, B, C)$, leaves the transfer function

$$
G(t, s)=C(t)\left(s I_{n}-A(t)\right)^{-1} B(t)=C(0)\left(s I_{n}-A(0)\right)^{-1} B(0)
$$

of the system invariant. We therefore term these flows isodynamical and a more systematic analysis of such flows is given in [9]. Obviously, these flows leave the eigenvalues of $A(t)$ invariant and in fact generalize the class of isospectral flows on matrices, obtained by letting $B=0, C=0$; see, e.g., [1], [3], and the references therein.

Simulations. Figures $4(\mathrm{a})-(\mathrm{c})$ show the evolution of the system matrices $(A, B, C)$ using this algorithm. In this example, the starting matrices are chosen to be

$$
A=\left[\begin{array}{ccc}
-3 & 0 & 0 \\
0 & -2 & 0 \\
0 & 0 & -1
\end{array}\right], \quad B=\left[\begin{array}{l}
2 \\
3 \\
1
\end{array}\right], \quad C^{\prime}=\left[\begin{array}{l}
2 \\
1 \\
3
\end{array}\right]
$$

and after ten "time intervals" the gramians are equal to three significant figures.

A similar "isodynamical flow approach" works also for obtaining diagonal balanced realizations. Here we consider the weighted cost function

$$
\Phi_{N}: \mathcal{R}_{G} \rightarrow \Re
$$

$$
\Phi_{N}(A, B, C)=\frac{1}{2} \operatorname{tr}\left[N\left(W_{c}(A, B)+W_{o}(A, C)\right)\right]
$$

for a real diagonal matrix $N$.

THEOREM 3.5. Let $\Phi_{N}: \mathcal{R}_{G} \rightarrow \Re$ be the objective function defined by (3.14) for $N=\frac{1}{2} \operatorname{diag}\left(\lambda_{1}, \ldots, \lambda_{n}\right), \lambda_{1}>\cdots>\lambda_{n}>0$.

(a) The gradient flow

$$
\left(\dot{A}=-\operatorname{grad}_{A} \Phi_{N}(A, B, C), \dot{B}=-\operatorname{grad}_{B} \Phi_{N}(A, B, C), \dot{C}=-\operatorname{grad}_{C} \Phi_{N}(A, B, C)\right)
$$

of $\Phi_{N}$ with respect to the normal Riemannian metric on $\mathcal{R}_{G}$ is

$$
\begin{aligned}
& \dot{A}=\left[A, N W_{o}(A, C)-W_{c}(A, B) N\right] \\
& \dot{B}=\left(N W_{o}(A, C)-W_{c}(A, B) N\right) B \\
& \dot{C}=C\left(W_{c}(A, B) N-N W_{o}(A, C)\right) .
\end{aligned}
$$

For every initial condition $(A(0), B(0), C(0)) \in \mathcal{R}_{G}$, the solution $(A(t), B(t), C(t)) \in$ $\mathcal{R}_{G}$ of (3.15) exists for all $t \geq 0$ and converges for $t \rightarrow+\infty$ to a diagonal balanced realization $(\bar{A}, \bar{B}, \bar{C})$ of $G(s)$, i.e., $W_{c}(\bar{A}, \bar{B})=W_{o}(\bar{A}, \bar{C})=$ diagonal.

(b) Suppose that the singular values of the Hankel of $(A, B, C)$ are distinct. Then (3.15) has exactly $2^{n}$ locally asymptotically stable equilibrium points $(\bar{A}, \bar{B}, \bar{C})$, characterized by $W_{c}(\bar{A}, \bar{B})=W_{o}(\bar{A}, \bar{C})=$ diagonal, with the diagonal elements in the reverse order to that of $N$. All other equilibria are unstable.

(c) The transfer function of every solution $(A(t), B(t), C(t))$ of $(3.15)$ is independent of $t$.

Proof. The proof runs similarly to that of Theorem 3.4, now applying Lemma 3.3 for $N=\frac{1}{2} \operatorname{diag}\left(\lambda_{1}, \ldots, \lambda_{n}\right)$ and using Proposition A.3. The only points we must check are that the equilibria of (3.15) are just the diagonal balanced realizations and 

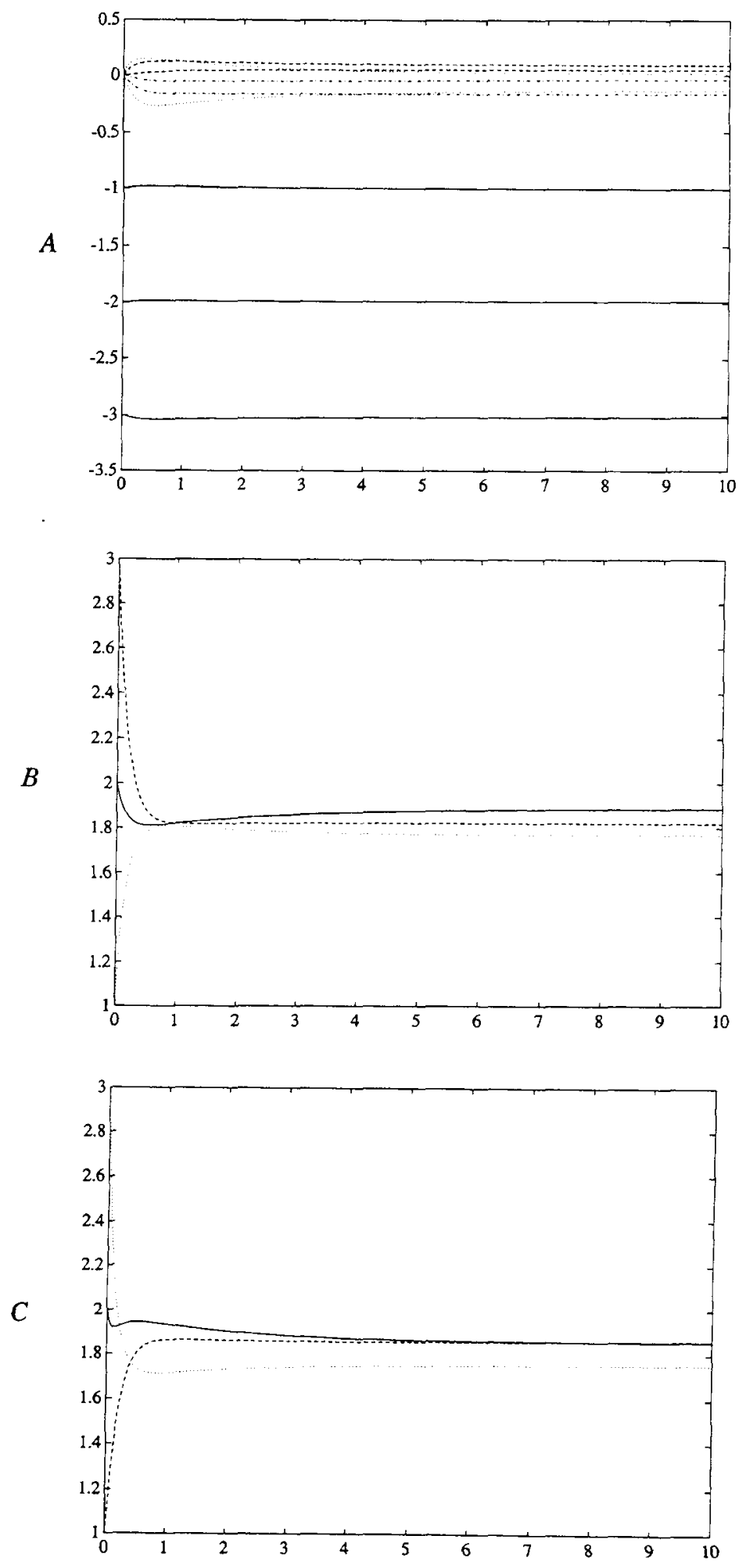

FIG. 4. Evolution of the system matrices $(A, B, C)$. 
their stability. But the equilibria of (3.15) are the critical points of $\Phi_{N}: \mathcal{R}_{G} \rightarrow \Re$ and hence correspond to those of $\Phi_{N} \circ \sigma: G L(n, \Re) \rightarrow \Re$. The result now follows from Lemma 2.6, and Theorem 2.7.

Simulations. Figure 5 shows the evolution of the matrices $(A, B, C)$ for $(3.15)$, with starting condition given in (3.13), and $N=\operatorname{diag}(3,2,1)$. After 30 "time intervals" the solution gives

$$
W_{o}=\left[\begin{array}{ccc}
2.7720 & 0 & 0 \\
0 & 0.1367 & 0.0214 \\
0 & 0.0214 & 0.0048
\end{array}\right], \quad W_{\mathrm{c}}=\left[\begin{array}{ccc}
2.7750 & 0 & 0 \\
0 & 0.1367 & 0.0212 \\
0 & 0.0212 & 0.0067
\end{array}\right]
$$

as opposed to the true balanced solution $W_{o}=W_{c}=\operatorname{diag}(0.0021,0.1401,2.7744)$. The convergence in this case can be expected to be slow because the smallest Hankel singular value is near zero.

4. Application to SVD. The common linear algebra problem of SVD can be solved using differential equations. Gradient flow solutions for SVD have been studied in [3], [4], [6], and [7]. Here we consider SVD to be a special case of the balanced realization task.

THEOREM 4.1. Given an $m \times n$ matrix $H$ of rank $r$ with distinct singular values $\sigma_{1}>\cdots>\sigma_{r}$. Let $N$ be an $r \times r$ diagonal matrix with distinct positive diagonal entries. Let $X_{0} \in \Re^{m \times r}$ and $Y_{0} \in \Re^{r \times n}$ be matrices of full rank $r$ such that $H=X_{0} Y_{0}$. Then the solution $(X(t), Y(t))$ of

$$
\begin{array}{cl}
\dot{X}=X\left(N Y Y^{\prime}-X^{\prime} X N\right), & X(0)=X_{0}, \\
\dot{Y}=\left(X^{\prime} X N-N Y Y^{\prime}\right) Y, & Y(0)=Y_{0}
\end{array}
$$

exists for all $t \geq 0$ and satisfies $H=X(t) Y(t)$ for all $t \geq 0$. The solution $(X(t), Y(t))$ converges to $\left(X_{\infty}, Y_{\infty}\right)$ such that $H=X_{\infty} Y_{\infty}$ and $X_{\infty}^{\prime} X_{\infty}=Y_{\infty} Y_{\infty}^{\prime}=D=$ diagonal. Moreover, there are $2^{r}$ stable equilibria that have the diagonal elements of $D$ in reverse order to those of $N$. All other equilibrium points are unstable.

Furthermore, this factorization yields $H=U S V$, where $U=X_{\infty} D^{-1 / 2}, S=D$, $V=D^{-1 / 2} Y_{\infty}, U^{\prime} U=I, V V^{\prime}=I$.

Proof. In Theorem 3.5, set $A=0$ and let $B, C$ be full rank matrices. Clearly, $(A, B, C)$ is controllable and observable. Then $W_{c}=B^{\prime} B, W_{o}=C C^{\prime}$ and (3.15) is equivalent to

$$
\dot{B}=-\left(C C^{\prime} N-N B^{\prime} B\right) B, \quad \dot{C}=C\left(C C^{\prime} N-N B^{\prime} B\right) .
$$

The equilibria of (4.2) are characterized by $B^{\prime} B=C C^{\prime}=$ diagonal, and the stable equilibria are such that $B^{\prime} B$ is in reverse order to $N$.

As (4.2) preserves the transfer function, $C B$ is constant. Hence (4.1) with $X=C$ and $Y=B$ converges to a diagonal balanced matrix factorization $H=X_{\infty} Y_{\infty}$. By choosing $U=X_{\infty} D^{-1 / 2}, S=D, V=D^{-1 / 2} Y_{\infty}$, then

$$
U S V=X_{\infty} D^{-1 / 2} D D^{-1 / 2} Y_{\infty}=X_{\infty} Y_{\infty}=H, \quad U^{\prime} U=D^{-1 / 2} X_{\infty}^{\prime} X_{\infty} D^{-1 / 2}=I,
$$

and $V V^{\prime}=D^{-1 / 2} Y_{\infty} Y_{\infty}^{\prime} D^{-1 / 2}=I$. The full singular value decomposition can be obtained by extending $U$ and $V$ to make them orthogonal. 

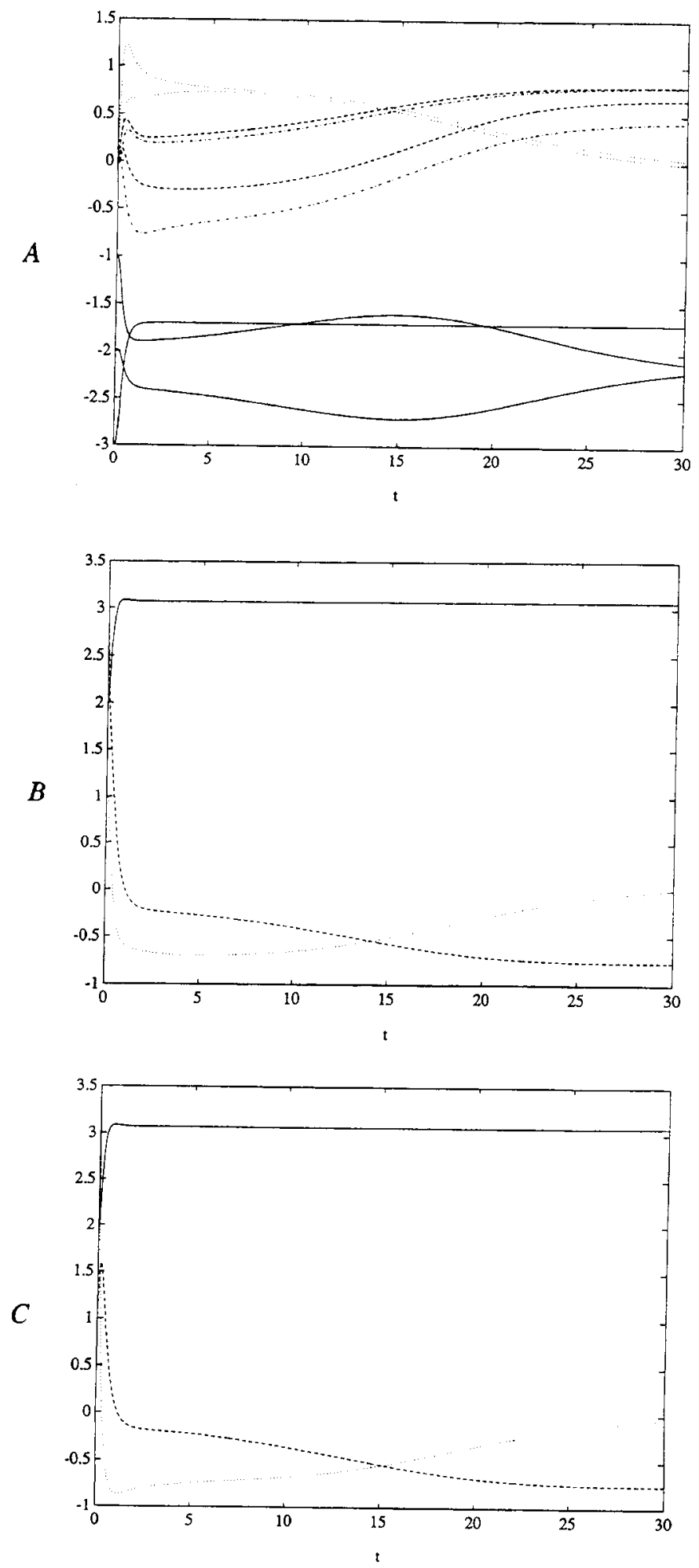

FIG. 5. Evolution of the diagonalizing system matrices $(A, B, C)$. 
5. Conclusions. There are a number of distinct ODEs that evolve to give the solution to the task of finding a balanced realization of a system or to the task of finding the SVD of a matrix. Each differential equation has distinct transient behaviour but all have exponential convergence rates of the factors. The dynamical systems for balancing gramains that were investigated evolve on either spaces of state coordinate transformation matrices $T$, its square $P=T^{\prime} T$, or on manifolds of the actual system matrices $(A, B, C)$. Similar equations for SVD are studied and are a special case of the balancing equations. Different convergence properties make some algorithms more attractive in certain problem settings. These solution methods may be useful when using analog or parallel computers.

Appendix. Riemannian metrics and gradient flows. Let $M$ be a smooth manifold and let $T M$ and $T^{*} M$ denote its tangent and cotangent bundle, respectively. A Riemannian metric on $M$ is a family of nondegenerate inner products $<,\rangle_{x}$, defined on each tangent space $T_{x} M$, such that $\left\langle,>_{x}\right.$ depends smoothly on $x \in M$. Any (nondegenerate) inner product on $\Re^{n}$ also defines a Riemannian metric on $\Re^{n}$ (but not conversely) and thus induces a Riemannian metric on every submanifold $M$ of $\Re^{n}$.

Let $\Phi: M \rightarrow \Re$ be a smooth function defined on the manifold $M$ and let $D \Phi$ : $M \rightarrow T^{*} M$ denote the differential, i.e., a section of the cotangent bundle $T^{*} M$. To define the gradient vector field of $\Phi$, we fix a Riemannian metric $<,>$ on $M$. The gradient $\nabla \Phi$ of $\Phi$ is then characterized by the following properties:

Compatibility condition (a). $D \Phi(x) \xi=\langle\nabla \Phi(x), \xi\rangle$ for all $\xi \in T_{x} M$.

Tangency condition (b). $\nabla \Phi(x) \in T_{x} M$ for all $x \in M$.

The following result is well known.

PROPOSITION A.1. There exists a uniquely determined vector field $\nabla \Phi$ on $M$ such that (a) and (b) hold. $\nabla \Phi$ is called the gradient vector field of $\Phi$.

Note that the gradient vector field depends on the choice of the Riemannian metric; changing the metric will also change the gradient.

It follows immediately from the definition of $\nabla \Phi$ that the equilibria of the differential equation

$$
\dot{x}(t)=-\nabla \Phi(x(t))
$$

are precisely the critical points of $\Phi$. Moreover, the linearization of the gradient flow (A.1) around each equilibrium point is given by the Hessian of $\Phi$ and thus has only real eigenvalues.

For any solution of (A.1),

$$
\frac{d}{d t} \Phi(x(t))=-\|\nabla \Phi(x(t))\|^{2}
$$

and therefore $\Phi(x(t))$ is monotonically decreasing. The following standard result is often used in this paper.

Proposttion A.2. Let $\Phi: M \rightarrow \Re$ be a smooth function on a Riemannian manifold with compact sublevel sets, i.e., for all $c \in \Re$ the sublevel set $\{x \in M \mid \Phi(x) \leq$ c\} is a compact subset of $M$. Then every solution $x(t) \in M$ of the gradient flow (A.1) on $M$ exists for all $t \geq 0$. Furthermore, $x(t)$ converges to a connected component of the set of critical points of $\Phi$ as $t \rightarrow+\infty$.

Note that the condition of the proposition is automatically satisfied if $M$ is compact. Moreover, in suitable local coordinates of $M$, the linearization of the gradient flow (A.1) around each equilibrium point has only real eigenvalues. 
Let $M$ be a smooth manifold and let $\Phi: M \rightarrow \Re$ be a smooth function. Let $C(\Phi) \subset M$ denote the set of all critical points of $\Phi$. We say $\Phi$ is a Morse-Bott function provided the following three conditions (i), (ii), (iii) are satisfied.

(i) $\Phi: M \rightarrow \Re$ has compact sublevel sets.

(ii) $C(\Phi)=\bigcup_{j=1}^{k} N_{j}$ with $N_{j}$ disjoint, closed, and connected submanifolds of $M$, such that $\Phi$ is constant on $N_{j}, j=1, \ldots, k$.

(iii) $\operatorname{Ker}(\operatorname{Hess} \Phi)_{x}=T_{x} N_{j}$ for all $x \in N_{j}, j=1, \ldots, k$.

Actually, the original definition of a Morse-Bott function also includes a global topological condition on the negative eigenspace bundle defined by the Hessian, but this condition is not relevant to us.

Recall that the $\omega$-limit set $L_{\omega}(x)$ of a point $x \in M$ for a vector field $X$ on $M$ is the set of points of the form $\lim _{n \rightarrow \infty} \phi_{t_{n}}(x)$, where $\left(\phi_{t}\right)$ is the flow of $X$ and $t_{n} \rightarrow+\infty$. Similarly, the $\alpha$-limit set $L_{\alpha}(x)$ is defined by letting $t_{n} \rightarrow-\infty$ instead of $+\infty$.

Proposition A.3.

(a) Suppose $\Phi: M \rightarrow \Re$ has isolated critical points. Then $L_{\omega}(x), x \in M$, consists of a single critical point. Therefore every solution of the gradient flow (A.1) converges for $t \rightarrow+\infty$ to a critical point of $\Phi$.

(b) Let $\Phi: M \rightarrow \Re$ be a Morse-Bott function on a Riemannian manifold $M$. Then the $\omega$-limit set $L_{\omega}(x), x \in M$, for the gradient flow (A.1) is a single critical point of $\Phi$. Every solution of the gradient flow (A.1) converges as $t \rightarrow+\infty$ to an equilibrium point.

\section{REFERENCES}

[1] R. W. BRocketT, Dynamical systems that sort lists, diagonalize matrices and solve linear programming problems, Linear Algebra Appl., 146 (1991), pp. 79-91.

[2] U. HELMKE, Balanced realizations for linear systems: a variational approach, SIAM J. Control Optim., 31 (1993), pp. 1-15.

[3] U. HELMKE AND J. B. MOORE, Singular value decomposition via gradient and self-equivalent flows, Linear Algebra Appl., 169 (1992), pp. 223-248.

[4] J. E. PERKINS, U. HELMKE, AND J. B. MOORE, Balanced realizations via gradient flows, Systems and Control Letters, 14 (1990), pp. 369-377.

[5] J. IMAE, J. E. Perkins, AND J. B. MOORE, Towards time varying balanced realization via Riccati equations, Math. Control, Signals, Systems, 1992, pp. 313-326.

[6] S. T. SMITH, Dynamical systems that perform the singular value decomposition, Systems Control Letters, 16 (1991), pp. 319-328.

[7] M. T. CHU AND K. R. DRIESSEL, The projected gradient method for least squares matrix approximations with spectral constraints, SIAM J. Numer. Anal., 27 (1990), pp. 1050-1060.

[8] T. Kallath, Linear Systems, Prentice-Hall, Englewood Cliffs, NJ, 1980.

[9] U. Helmke and J. B. Moore, Optimization and Dynamical Systems, Springer-Verlag, New York, 1993.

[10] B. C. MOORE, Principal component analysis in linear systems: controllability, observability, and model reduction, IEEE Trans. Automat. Control, AC-26 (1981), pp. 17-32.

[11] A. J. Lavb, M. T. Heath, C. C. Paige, AND R. C. Ward, Computation of system balancing transformations and other applications of simultaneous diagonalization algorithms, IEEE Trans. Automat. Control, AC-32 (1987), pp. 115-121.

[12] M. C. IRwin, Smooth Dynamical Systems, Academic Press, London, 1980. 